\title{
Illumination Normalized Face Image for Face Recognition
}

\author{
Jaepil Ko, Eunju Kim, and Heyran Byun \\ Dept. of Computer Science, Yonsei Univ. \\ 134, Shinchon-dong Sudaemoon-ku, Seoul, 120-749, Korea \\ \{nonezero, outframe, hrbyun\}@csai.yonsei.c.kr
}

\begin{abstract}
A small change in illumination produces large changes in appearance of face even when viewed in fixed pose. It makes face recognition more difficult to handle. To deal with this problem, we introduce a simple and practical method based on the multiple regression model, we call it ICR (Illumination Compensation based on the Multiple $\underline{\mathrm{Re}}$ gression Model). We can get the illumination-normalized image of an input image by ICR. To show the improvement of recognition performance with ICR, we applied ICR as a preprocessing step. We achieved better result with the method in preprocessing point of view when we used a popular technique, PCA, on a public database and our database.
\end{abstract}

\section{Introduction}

The visual recognition system suffers from the different appearances of objects according to the illumination conditions [1]. Especially face images are highly sensitive to the variations in illumination conditions so small change in illumination produces large changes in appearance of face [2]. That makes face recognition/verification problem more difficult to handle. The FERRET test report shows that the performance significantly drops in the case of the illumination changes $[3,4]$. Until now many face recognition methods have been proposed and there are several methods for dealing with illumination problem. For details, reader should consult recent survey paper [5].

The first approach to handle the effects results from illumination changes is constructing illumination model from several images acquired under different illumination condition [6]. The representative method, the illumination cone model that can deal shadow and multiple lighting sources, is introduced by [7,8]. This approach is not practical in smart card application, which can just memorize one or two presentations (prototypes) of a person and to construct the cone model for a person it needs wellcontrolled image capturing circumstances. The standard answer for the problem with variable lightings, the second approach, is to extract illumination invariant features, such as edges, corner, and contour, which is often considered as the basic image representation but these are insufficient to contain useful information for recognition. Furthermore, edges are susceptible to the illumination conditions for complex object 
and when the image has cluttered background. Instead of edge-based description, image-based description is preferred in face recognition system. The method use lowdimensional representation of image by subspace technique such as Eighenfaces and Fisherfaces [9]. In the above case, with the assumption that the first few principal components are strongly involved in illumination effects, discarding the first three principal components improves recognition performance under illumination changes [10]. However, the performance is not improved on images captured under normal illumination. Because in the case of normal lighted images discarding the first three components could also eliminate important information for recognition. Another eigenspace method was developed by [11]. The major idea was to incorporate a set of gradient based filter banks into the eigenspace recognition framework. It might strongly depend on gradient operator being charge of illumination variations. Without loosing important information in an image itself, the SSFS (symmetric shape-fromshading) algorithm as a tool to obtain illumination-normalized prototype image, which is based on shape-from-shading assuming just one image is available was proposed by [12].

In this paper, we describe more simple and practical algorithm for getting illumination-compensated face image by applying the illumination compensation method based on the multiple regression model for finding the best-fit intensity plane.

This paper is organized as follows. In the next section, we overview multiple regression model in brief. Section 3, we describe simple illumination compensation algorithm, we call it ICR (Illumination Compensation based on the multiple Regression model), for face image. The experimental results are shown in section 4. Finally, in section 5 conclusions are drawn.

\section{Multiple Regression Model}

In this section, we will give a brief overview of MRM (multiple regression model) well known technique in statistics. For details of MRM see the book [13]. MRM is the linear regression model for multivariate case.

The multiple regression model can be written as

$$
\mathbf{Y}=\mathbf{X} \boldsymbol{\beta}+\mathbf{e}
$$

where $\mathbf{Y}$ is an $n \times 1$ response vector, $\mathbf{X}$ is an $n x(k+1)$ matrix for an $k$ input variables and $n$ samples, and $\mathbf{e}$ is an $n \times 1$ random error vector that we shall assume is normally distributed with mean 0 and variance $\sigma^{2}$.

The parameters $\boldsymbol{\beta}$ and $\sigma^{2}$ must be estimated from the samples. If we let $\mathbf{B}^{\mathrm{T}}=\left[\mathrm{B}_{0} \mathrm{~B}_{1} \cdots \mathrm{B}_{\mathrm{k}}\right]$ of the least square estimator of the $\boldsymbol{\beta}$. Then the least square estimator $\mathbf{B}$ are given by

$$
\mathbf{B}=\left(\mathbf{X}^{\mathrm{T}} \mathbf{X}\right)^{-1} \mathbf{X}^{\mathrm{T}} \mathbf{Y}
$$

In finding the best-fit intensity plane, input variable is the coordinate of each pixel and response is the intensity value of the location and the number of samples are num- 
ber of pixels of the image. After estimating parameters, we can get a new intensity value of the location. That is the best-fit intensity plane of the image. The next section will give you for details.

\section{Illumination Compensation Based on the Multiple Regression Model (ICR)}

We try to get illumination- compensated face image by itself, which is similar to the stored prototype image when the input face image is captured under different illumination conditions without any illumination model. We assume that small set of face images is available which is acquired under a single ambient light. That is practical assumption. Because in office circumstance we can easily imagine that the window sided face image is brighter than that of the other side.

To get illumination- compensated face image, we first find the best-fit intensity plane of an input image. The best-fit intensity plane can be found by the multiple regression model described in section 2 . We start with a face image whose dimension is $q$ from $n \times m$ face image pixels.

$$
\mathbf{x}=\left[\mathrm{x}_{0}, \mathrm{x}_{1}, \cdots, \mathrm{x}_{\mathrm{q}-1}\right]^{\mathrm{T}}
$$

then, we generate $q$ samples for the regression model.

$$
\begin{aligned}
& \mathbf{z}_{\mathrm{k}}=\left[\mathrm{i}, \mathrm{j}, \mathrm{x}_{\mathrm{k}}\right]^{\mathrm{T}}, \mathrm{k}=\mathrm{i} \times \mathrm{m}+\mathrm{j} \\
& \mathrm{i}=0,1, \cdots, \mathrm{n}-1, \mathrm{j}=0,1, \cdots, \mathrm{m}-1
\end{aligned}
$$

where the $i$ and $j$ are input values and $\mathrm{x}_{\mathrm{k}}$ is response value for the regression model. After applying samples $\mathbf{z}_{\mathrm{k}}$ to the regression, we can get the best-fit intensity plane:

$$
\mathbf{y}=\left[\mathrm{y}_{0}, \mathrm{y}_{1}, \cdots, \mathrm{y}_{\mathrm{q}-1}\right]^{\mathrm{T}}
$$

The center value in the best-fit intensity plane $y_{c}=\left[\max \left(y_{i}\right)-\min \left(y_{j}\right)\right] / 2, i<j<q$, is computed. The next step is that we reverse the intensity plane against its center value:

$$
\mathbf{y}^{\prime}=\left[\mathrm{y}_{\mathrm{c}}-\mathrm{y}_{0}, \mathrm{y}_{\mathrm{c}}-\mathrm{y}_{1}, \cdots, \mathrm{y}_{\mathrm{c}}-\mathrm{y}_{\mathrm{q}-1}\right]^{\mathrm{T}}
$$

Finally, we can get illumination- compensated face image by adding the original input image $\mathbf{x}$ and the adjusted intensity image $\mathbf{y}^{\prime}$.

The Fig. 1 shows an original input image, the best-fit intensity plane, adjusted intensity plane, and illumination- compensated image by applying our method. The final image appears a little bit uniform intensity preserving its relative intensity.

To compare the existing preprocessing techniques such as gamma correction and histogram equalization are shown in the Fig. 2. 


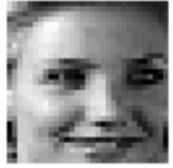

(a)

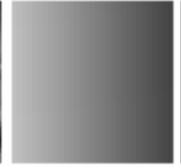

(b)

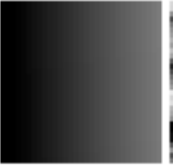

(c)

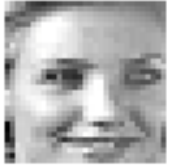

(d)

Fig. 1. (a) input image, (b) the best-fit intensity plane, (c) adjusted intensity plane, (d) final result from adding (a) and (c)

Segmenting an object in a gradient image is very difficult problem in threshold techniques. The box object in the image can be easily segmented by a single threshold in the illumination-compensated image. In the case of gamma correction, the gradient of the image was not changed when the global intensity became bright. The image with histogram equalization, it made worse segment the box object by striking the gradient of the input image.

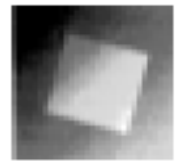

(a)

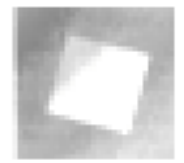

(d)

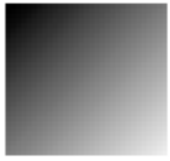

(b)

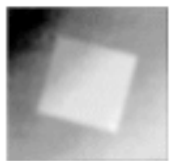

(e)

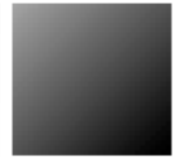

(c)

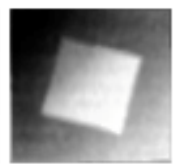

(f)

Fig. 2. (a) original input image, (b) the best-fit intensity plane of (a), (c) adjusted the best-fit intensity plane, (d) illumination-compensated image of (a), (e) image with gamma correction, (f) histogram equalized image of (a)

\section{$4 \quad$ Experimental Results}

In this section, we show that the performance of ICR in preprocessing point of view by comparing the histogram equalization widely used for normalizing illumination effect in face recognition system. We also compared the standard technique that drops the first three components in PCA for mitigating illumination effect [10].

All the face images were preprocessed to normalize geometry and to remove background. The preprocessing procedure is that we first manually located the centers of the eyes for translating, rotating and scaling the faces, and then applied the histogram equalization or ICR. The next step is to scale facial pixels to have zero mean and unit variance that is required for PCA inputs to improve the performance. In the last step, we masked the face image to remove the background and hair. 
We have applied ICR to both the Cambridge ORL face database and our database to demonstrate the improvement of face recognition performance. Our database contains 200 frontal face images for 200 individuals captured with single light source.

PCA was performed on all the images on each database and made a probe set by flipping the face image by turning right-side left. In our experiments, we applied the nearest neighbor classifier.

Four types of preprocessed face image whose size is $32 \times 32$ are shown in Fig. 3. The right part of the face image is darker than that of left side due to light source on the left hand. The difference between the both sides gets tight in (b) by histogram equalization. However, the gap between them is loosed by ICR in (c). We expected that this effect would improve recognition performance.

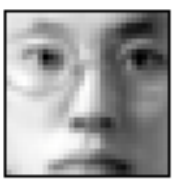

(a)

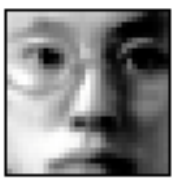

(b)

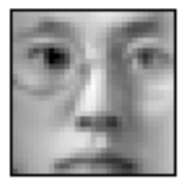

(c)

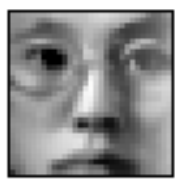

(d)

Fig. 3. (a) Original image, (b) histogram equalized image, (c) ICR processed image, (d) histogram equalization followed by ICR

First, we experimented the effect of discarding the first few components in PCA on the ORL database. In the ORL database, in the case of applying ICR the recognition performance was better when it included the first component. However, Original and histogram equalized image showed better results without the first component.According to the result, we could carefully say that ICR efficiently removes illumination-effect contained in the first few principle components. Table 1 shows the results.

Table 1. The recognition performance on the ORL database with rank 1, original (Org), histogram equalization (HE), ICR, and histogram equalization followed by ICR (HE\&ICR). The row was tested without the first $\mathrm{n}$ principal components

\begin{tabular}{c|c|c|c|c} 
& Org & HE & ICR & HE \& ICR \\
\hline w/o 0 & 0.63 & 0.49 & $\mathbf{0 . 8 0}$ & $\mathbf{0 . 7 4}$ \\
\hline w/o 1 & $\mathbf{0 . 6 6}$ & $\mathbf{0 . 7 7}$ & 0.76 & 0.63 \\
\hline W/o 2 & 0.65 & 0.75 & 0.69 & 0.56 \\
\hline w/o 3 & 0.53 & 0.69 & 0.65 & 0.46 \\
\hline w/o 4 & 0.47 & 0.65 & 0.60 & 0.36
\end{tabular}

Second, we collected the best results by changing the number of principal components and listed in Table 2, 3 on the ORL database and on our database respectively.

We had the same result on both the ORL database and our database. The proposed technique, ICR, showed the best result among them but we could not achieve the same result on the subset including face images just related to the light conditions in the Yale database. On the Yale database, the HE\&ICR showed better result. It means that histogram equalization is still useful when the illumination situation is worse than normal case. In addition, on the Yale database the performance of the techniques with 
ICR is remarkable comparing to the others without ICR. Fig. 4 shows the results. We tested rank 1 success rate as removing the first eigenvectors. ICR, ICR followed by HE and HE followed by ICR techniques applying our proposed method show better result than ORG and HE techniques. In the case of ORG and HE, they achieved best result when it removed the first two or three eigenvectors respectively. The techniques applying both ICR and HE show the best results when they used the first eigenvector. It means that our proposed method is alleviating illumination effects like eigentechnique discarding the first $\mathrm{n}$ eigenvectors. Moreover, it does not remove any important information for recognition.

Table 2. The recognition performance on the ORL database from rank 1 to rank 10 . For each rank, the first row is success rate and the second row is the success number of faces among 400 faces

\begin{tabular}{c|c|c|c|c} 
& Org & HE & ICR & HE\&ICR \\
\hline \multirow{2}{*}{ rank1 } & 0.66 & 0.77 & $\mathbf{0 . 8 0}$ & 0.74 \\
\cline { 2 - 5 } & 265 & 307 & 318 & 294 \\
\hline \multirow{2}{*}{ rank2 } & 0.73 & 0.83 & $\mathbf{0 . 8 5}$ & 0.80 \\
\cline { 2 - 5 } & 292 & 332 & 340 & 320 \\
\hline \multirow{2}{*}{ rank3 } & 0.78 & 0.85 & $\mathbf{0 . 8 7}$ & 0.85 \\
\cline { 2 - 5 } & 310 & 341 & 348 & 338 \\
\hline \multirow{2}{*}{ rank4 } & 0.80 & 0.87 & $\mathbf{0 . 8 9}$ & 0.87 \\
\cline { 2 - 5 } & 321 & 349 & 355 & 348 \\
\hline \multirow{2}{*}{$\operatorname{rank5}$} & 0.82 & 0.88 & $\mathbf{0 . 9 0}$ & 0.88 \\
\cline { 2 - 5 } & 327 & 352 & 360 & 353 \\
\hline \multirow{2}{*}{ rank10 } & 0.87 & 0.92 & $\mathbf{0 . 9 5}$ & 0.93 \\
\cline { 2 - 5 } & 347 & 368 & 381 & 372
\end{tabular}

Table 3. The recognition performance on our database from rank 1 to rank 10 . For each rank, the first row is success rate and the second row is the success number of faces among 200 faces

\begin{tabular}{c|c|c|c|c} 
& Org & HE & ICR & HE \& ICR \\
\hline \multirow{2}{*}{ rank1 } & 0.47 & 0.60 & $\mathbf{0 . 7 6}$ & 0.62 \\
\cline { 2 - 5 } & 93 & 119 & 151 & 123 \\
\hline \multirow{2}{*}{ rank2 } & 0.55 & 0.65 & $\mathbf{0 . 8 5}$ & 0.72 \\
\cline { 2 - 5 } & 109 & 130 & 170 & 144 \\
\hline \multirow{2}{*}{ rank3 } & 0.57 & 0.71 & $\mathbf{0 . 9 0}$ & 0.77 \\
\cline { 2 - 5 } & 113 & 141 & 179 & 153 \\
\hline \multirow{2}{*}{ rank4 } & 0.60 & 0.74 & $\mathbf{0 . 9 1}$ & 0.81 \\
\cline { 2 - 5 } & 119 & 147 & 182 & 162 \\
\hline \multirow{2}{*}{ rank5 } & 0.64 & 0.76 & $\mathbf{0 . 9 2}$ & 0.84 \\
\cline { 2 - 5 } & 128 & 152 & 184 & 167 \\
\hline \multirow{2}{*}{ rank10 } & 0.72 & 0.82 & $\mathbf{0 . 9 4}$ & 0.87 \\
\cline { 2 - 5 } & 143 & 163 & 188 & 174
\end{tabular}




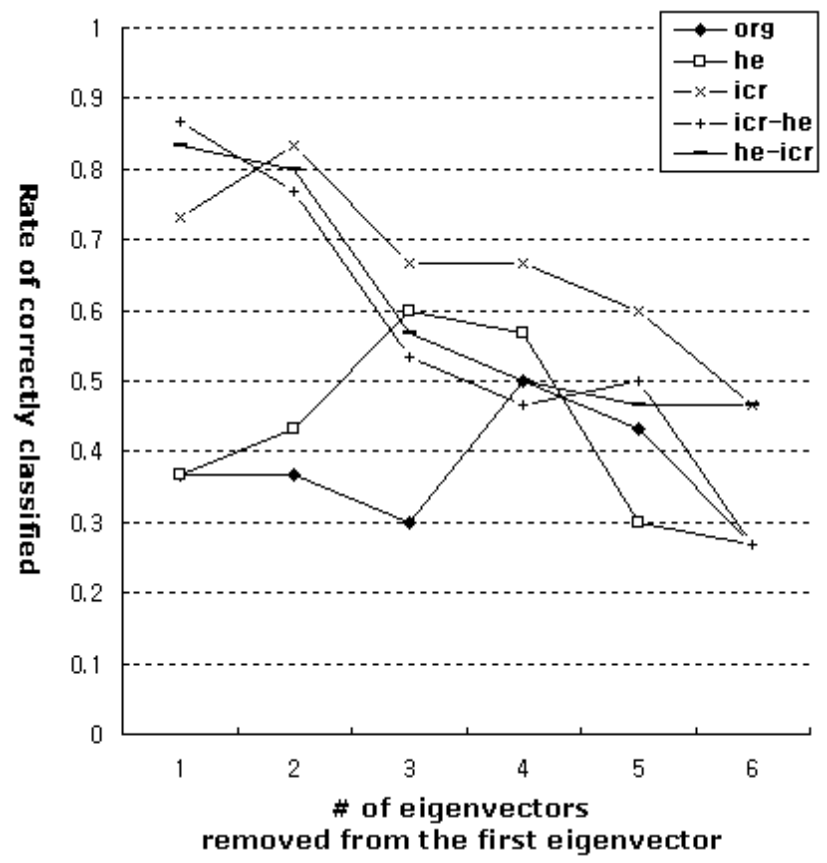

Fig. 4. Comparison of success rate according to the preprocessing technique. The techniques applying our proposed method outperform when they use the first eigenvector

\section{Conclusions}

Face images are highly sensitive to the variations in illumination conditions. We described a simple and practical method, ICR, to get an illumination- compensated image. The method does not need to construct illumination model from several images, also it does not remove any important information for recognition by discarding the first $n$ principal components.

We showed experimentally that the possibility of the normalized face image preprocessed by ICR could be used to alleviate illumination effect in face recognition system.

We think that the ICR can be one of the preprocessing techniques in face recognition system for its simplicity and effectiveness of dealing with illumination effect. However, we assumed that the light source is single and ambient. In future work, even thought it is a practical assumption we will extend experimentation by applying ICR locally to handle the situation that several light sources exist. 


\section{Acknowledgement}

This research was supported as a Brain Neuroinformatics Research Program sponsored by the Korean Ministry of Science and Technology (M1-0107-00-0008).

\section{References}

1. Michael J. Tarr, Daniel Kersten, Heinrich H. Bulthoff, : Why the visual recognition system might encode the effects of illumination, Pattern Recognition (1998)

2. Yael Adini, Yael Moses, and Shimon Ullman, : Face Reconition: The problem of Compensating for Changes in Illumination Direction, IEEE Trans. on PAMI Vol. 19, No. 7 (1997) 721-732

3. P. J. Phillips, H. Moon, P. Rauss, and S. A. Rizvi. : The FERET Evaluation Methodology for Face-Recognition Algorithms. IEEE Conference on CVPR, Puerto Rico (1997) 137-143

4. S. Rizvi, P. Phillips, and H. Moon. : The FERET verication testing protocol for face recognition algorithms. IEEE Conference on Automatic Face- and GestureRecognition (1998) 48-53

5. R. Chellappa and W. Zhao, : Face Recognition: A Literature Survey. ACM Journal of Computing Surveys (2000)

6. A. Yuille, D. Snow, R. Epstein, P. Belhumeur, : Determining Generative Models of Objects Under Varying Illumination: Shape and Albedo from Multiple Images Using SVD and Integrability, International Journal of Computer Vision, 35 (3)(1999) 203-222

7. P. N. Belhumeur and D. J. Kriegman. : What is the set of images of an object under all possible lighting conditions?, IEEE Conference on CVPR (1996)

8. Athinodoros S. Georghiades, David J. Kriegman, Peter N. Belhumeur, : Illumination Cones for Recognition Under Variable Lighting: Faces, IEEE Conference on CVPR (1998) 52-58

9. M.Turk and A. Pentland, : Eigenfaces for recognition. Journal of Cognitive Neuroscience, Vol 3 (1991)

10. V. Belhumeur, J. Hespanha, and D. Kriegman. : Eigenfaces vs. fisherfaces: Recognition using class specific linear projection. IEEE Trans. on PAMI (1997) 711720

11. Bischof, H.; Wildenauer, H.; Leonardis, A.: Illumination insensitive eigenspaces, IEEE Conference on Computer Vision, Vol. 1 (2001) 233-238

12. Wen Yi Zhao; Chellappa, R. : Illumination-Insensitive Face Recognition Using Symmetric Shape-from-Shading, IEEE Conference on CVPR, Vol. 1, (2000) 286-293

13. S. M. Ross, : Introduction to Probability and Statistics for Engineers and Scientists, Wiley, New York (1987) 